\title{
ARTICLES
}

\section{Coherent production of single pions and $\rho$ mesons in charged-current interactions of neutrinos and antineutrinos on neon nuclei at the Fermilab Tevatron}

\author{
S. Willocq, ${ }^{o}$ M. Aderholz, ${ }^{\lambda}$ H. Akbari, ${ }^{o,(a)}$ P. P. Allport, ${ }^{\mu,(b)}$ S. K. Badyal, ${ }^{\kappa}$ \\ H. C. Ballagh, ${ }^{\alpha}$ M. Barth, ${ }^{\gamma}$ H. H. Bingham,$^{\alpha}$ E. B. Brucker, ${ }^{\xi}$ R. A. Burnstein, ${ }^{\iota}$ \\ R. J. Cence, ${ }^{\eta}$ T. K. Chatterjee, ${ }^{\epsilon}$ E. F. Clayton, ${ }^{\theta}$ G. Corrigan ${ }^{\mu}$ D. De Prospo, ${ }^{\nu}$ \\ Devanand, ${ }^{\kappa}$ E. De Wolf, ${ }^{\gamma,}(\mathrm{c})$ P. J. W. Faulkner, ${ }^{\beta}$ H. Foeth ${ }^{\delta}$ W. B. Fretter, ${ }^{\alpha,(d)}$ \\ V. K. Gupta, ${ }^{\kappa}$ J. Hanlon, ${ }^{\zeta}$ G. Harigel, ${ }^{\delta}$ F. A. Harris,${ }^{\eta}$ P. Jacques, ${ }^{\nu}$ V. Jain,${ }^{\eta,(e)}$ \\ G. T. Jones, ${ }^{\beta}$ M. D. Jones, ${ }^{\eta}$ T. Kafka, ${ }^{o}$ M. Kalelkar, ${ }^{\nu}$ J. M. Kohli, ${ }^{\epsilon}$ \\ E. L. Koller, ${ }^{\xi}$ R. J. Krawiec, ${ }^{\beta}$ M. Lauko, ${ }^{\nu}$ J. E. Lys, ${ }^{\alpha}$ P. Marage, ${ }^{\gamma}$ \\ R. H. Milburn, ${ }^{\circ}$ I. S. Mittra, ${ }^{\epsilon}$ M. M. Mobayyen, ${ }^{\theta}$ J. Moreels, ${ }^{\gamma}$ \\ D. R. O. Morrison, ${ }^{\delta}$ G. Myatt, ${ }^{\mu}$ P. Nailor, ${ }^{\theta,(b)}$ R. Naon, ${ }^{\iota}$ A. Napier ${ }^{o}$ \\ D. Passmore, ${ }^{o}$ M. W. Peters,${ }^{\eta}$ V. Z. Peterson, ${ }^{\eta}$ R. Plano, ${ }^{\nu}$ N. K. Rao, ${ }^{\kappa}$ \\ H. A. Rubin, ${ }^{\iota}$ J. Sacton, ${ }^{\gamma}$ S. S. Sambyal,${ }^{\kappa}$ N. Schmitz,${ }^{\lambda}$ J. Schneps ${ }^{o}$ \\ J. B. Singh, ${ }^{\epsilon}$ S. Singh,$^{\epsilon}$ W. Smart ${ }^{\zeta}$ P. Stamer ${ }^{\nu,(f)}$ K. E. Varvell, ${ }^{\beta,(g)}$ \\ L. Verluyten, ${ }^{\gamma,(\mathrm{c})},(\mathrm{h})$ H. Wachsmuth, ${ }^{\delta}$ S. Wainstein, ${ }^{\theta}$ and G. P. Yost ${ }^{\alpha,(\mathrm{i})}$ \\ (E632 Collaboration) \\ a University of California, Berkeley, California 94720 \\ $\beta$ University of Birmingham, Birmingham B15 2TT, United Kingdom \\ $\gamma$ Inter-University Institute for High Energies (ULB-VUB), B-1050 Brussels, Belgium \\ ${ }^{\delta}$ CERN, CH-1211 Geneva 23, Switzerland \\ $\epsilon$ Panjab University, Chandigarh - 160014, India \\ $\varsigma$ Fermilab, Batavia, Illinois 60510 \\ $\eta$ University of Hawaii, Honolulu, Hawaii 96822 \\ ${ }^{\theta}$ Imperial College of Science, Technology and Medicine, London, SW7 2AZ, United Kingdom \\ ¿ Illinois Institute of Technology, Chicago, Illinois 60616 \\ $\kappa$ University of Jammu, Jammu - 180001, India \\ ${ }^{\lambda}$ Max-Planck-Institut für Physik, D-8000 München 40, Germany \\ ${ }^{\mu}$ Department of Nuclear Physics, Oxford University, Oxford, OX1 3RH, United Kingdom \\ $\nu$ Rutgers University, New Brunswick, New Jersey 08903 \\ $\xi$ Stevens Institute of Technology, Hoboken, New Jersey 07030 \\ - Tufts University, Medford, Massachusetts 02155
}

(Received 25 September 1992)

\begin{abstract}
The coherent production of $\pi$ and $\rho$ mesons in $\nu_{\mu}\left(\bar{\nu}_{\mu}\right)$-neon charged-current interactions has been studied using the Fermilab 15 -foot bubble chamber filled with a heavy $\mathrm{Ne}^{-\mathrm{H}_{2}} \mathrm{mix}$ and exposed to the Tevatron quadrupole triplet (anti)neutrino beam. The $\nu_{\mu}\left(\bar{\nu}_{\mu}\right)$ beam had an average energy of 80 $\mathrm{GeV}(70 \mathrm{GeV})$. From a sample corresponding to approximately 28000 charged-current interactions, net signals of $(53 \pm 9) \mu^{ \pm} \pi^{\mp}$ coherent events and $(19 \pm 7) \mu^{ \pm} \pi^{\mp} \pi^{0}$ coherent events are extracted. For $E>10 \mathrm{GeV}$, the coherent pion production cross section is determined to be $(3.2 \pm 0.7) \times 10^{-38}$ $\mathrm{cm}^{2}$ per neon nucleus whereas the coherent $\rho$ production cross section is $(2.1 \pm 0.8) \times 10^{-38} \mathrm{~cm}^{2}$ per neon nucleus. These cross sections and the kinematical characteristics of the coherent events at $|t|<0.1 \mathrm{GeV}^{2}$ are found to be in general agreement with the predictions of a model based on the hadron dominance and, in the pion case, on the partially conserved axial-vector current hypothesis. Also discussed is the coherent production of systems consisting of three pions.
\end{abstract}

PACS number(s): 13.15.Dk, 11.40.Ha, 12.40.Vv

\footnotetext{
(a) Now at CERN, Switzerland.

(b) Now at Cavendish Laboratory, Cambridge, U.K.

(c) Also at Universitaire Instelling Antwerpen, B-2610 Wilrijk, Belgium.

${ }^{(d)}$ Deceased.
}

\footnotetext{
(e) Now at Vanderbilt University, Nashville, TN 37235.

(f) At Seton Hall University, S. Orange, NJ 07079.

(g) Now at ANSTO, Menai NSW 2234, Australia.

(h) Now at Xeikon, B-2640 Mortsel, Belgium.

(i) Now at SSC Laboratory, Dallas, TX 75237.
} 


\section{INTRODUCTION}

The study of coherent neutrino and antineutrino interactions over the past ten years has provided much information on the structure of the weak hadronic current (see Ref. [1] for a comprehensive review). In particular, the study of single pion coherent production in both neutraland charged-current interactions has provided one of the rare tests of the partially conserved axial-vector current hypothesis (PCAC) at high energy [see Ref. [2] for a test of PCAC in inclusive (anti)neutrino-proton scattering].

The test of the PCAC hypothesis is based on Adler's theorem [3]: because of PCAC, the neutrino scattering cross section $\sigma\left(\nu_{\mu} \mathcal{N} \rightarrow \mu X\right)$ is proportional to the pion scattering cross section $\sigma(\pi \mathcal{N} \rightarrow X)$ for $Q^{2}=0\left(Q^{2}\right.$ is the negative of the square of the intermediate boson four-momentum).

For $Q^{2} \neq 0$, predictions are derived from the hadron dominance model [4]: the weak hadronic current may be viewed as a superposition of vector and axial-vector states, with the same quantum numbers as the weak intermediate boson. The weak vector current is thus dominated by the $\rho$ meson, as in the vector meson dominance model for electromagnetic interactions [5], and the vector part of the neutrino scattering cross section is related to the $\rho$ meson scattering cross section $\sigma(\rho \mathcal{N} \rightarrow X)$.

The weak axial-vector current is assumed to be dominated by the $a_{1}$ meson (chiral partner of the $\rho$ ) or, more likely, by nonresonant $\rho \pi$ states (see Refs. $[1,6-8]$ ). The longitudinal component of $\rho \pi$ or $a_{1}$ states is thus responsible for the scattering at $Q^{2}=0$; the pion contribution to the axial current plays only a negligible role [9].

In coherent interactions, the nucleus remains intact, and the scattering amplitude of the weak current on all the nucleons in the nucleus gives rise to maximum constructive interferences. The coherence condition implies that

$$
|\mathbf{k}| \lesssim 1 / R_{\mathcal{N}}
$$

where $k$ is the four-momentum transfer to the nucleus of radius $R_{\mathcal{N}}$. More precisely, the nucleus form factor implies a sharp falloff of the $|t|$ distribution $\left(t=-k^{2}\right)$. However, the kinematics of the reaction implies

$$
|t|>t_{\min } \simeq\left(\frac{Q^{2}+m^{2}}{2 \nu}\right)^{2},
$$

where $m$ is the mass of the hadronic state produced, and $\nu$ is the laboratory energy of the intermediate boson. Consequently, coherent interactions are mostly restricted to the low $Q^{2}$, high $\nu$ region, i.e., low Bjorken $x$.

The main goal of this paper is the study of the coherent production of both single pions and single $\rho$ mesons in (anti)neutrino charged-current interactions on neon nuclei (see Fig. 1) using the Fermilab 15-foot bubble chamber:

$$
\begin{aligned}
& \nu_{\mu}+\mathrm{Ne} \rightarrow \mu^{-}+\pi^{+}+\mathrm{Ne} \\
& \bar{\nu}_{\mu}+\mathrm{Ne} \rightarrow \mu^{+}+\pi^{-}+\mathrm{Ne} \\
& \nu_{\mu}+\mathrm{Ne} \rightarrow \mu^{-}+\rho^{+}+\mathrm{Ne} \\
& \bar{\nu}_{\mu}+\mathrm{Ne} \rightarrow \mu^{+}+\rho^{-}+\mathrm{Ne},
\end{aligned}
$$

a)

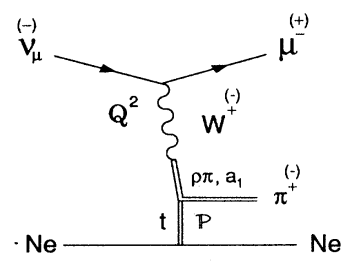

b)

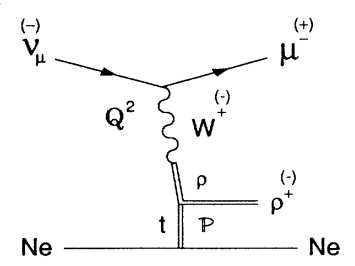

FIG. 1. Diagrams for the coherent production of (a) a single pion and (b) a single $\rho$ meson.

with $\rho^{ \pm} \rightarrow \pi^{ \pm} \pi^{0}$ and $\pi^{0} \rightarrow \gamma \gamma$. The results of our first study of reactions (1), using a significantly smaller data sample, were reported in Ref. [10]. There have been several studies of the coherent production of pions [11-18], and two bubble chamber experiments reported the observation of $\rho$ coherent production $[19,20]$. In addition to reactions (1) and (2), we have also investigated the coherent production of hadronic systems decaying into $(3 \pi)$ final states for which evidence has been reported by only one previous experiment [7]. All previous coherent production studies were carried out at lower neutrino beam energies whereas the present experiment extends the energy range up to $\sim 300 \mathrm{GeV}$.

The results are compared with the predictions for $\pi$ and $\rho$ meson coherent production based on the PCAC hypothesis and on the hadron dominance model. The Appendix gives the corresponding cross sections, including the effect of the assumption of a Regge $\rho \pi$ cut or $a_{1}$ pole dominance of the weak axial current. To compute the $\pi$-nucleus and $\rho$-nucleus elastic cross sections, we have used full relations derived from the Glauber model [21]. For comparison, we have also used approximated relations, obtained in the framework of the Glauber model by Belkov and Kopeliovich [6].

\section{EXPERIMENTAL PROCEDURE}

The data used in this analysis were collected during the 1985 and 1987-1988 data taking periods of the Fermilab Tevatron. The 15-foot bubble chamber was filled with a heavy $\mathrm{Ne}^{-} \mathrm{H}_{2}$ mixture, 75 mole percent neon during the 1985 run and 63 mole percent neon during the 1987-1988 run. The respective liquid densities were 0.71 $\mathrm{g} \mathrm{cm}^{-3}$ and $0.54 \mathrm{~g} \mathrm{~cm}^{-3}$, and the radiation lengths were $41.5 \mathrm{~cm}$ and $55.0 \mathrm{~cm}$. The bubble chamber was exposed to the quadrupole triplet (anti)neutrino beam which was produced by $800 \mathrm{GeV}$ protons from the Tevatron. The average $\nu_{\mu}\left(\bar{\nu}_{\mu}\right)$ beam energy was $80 \mathrm{GeV}(70 \mathrm{GeV})$ and the average $\nu_{\mu}\left(\bar{\nu}_{\mu}\right)$ event energy was $150 \mathrm{GeV}(110 \mathrm{GeV})$; see Fig. 2 for the calculated $\nu_{\mu}$ and $\bar{\nu}_{\mu}$ beam energy spectra [22]. The $\bar{\nu}_{\mu}$ to $\nu_{\mu}$ flux ratio was approximately 2:5. More details about the experimental conditions can be found in Ref. [23].

The bubble chamber (Fig. 3) was equipped with new arrays of proportional tubes: the internal picket fence (IPF) and the external muon identifier (EMI) [24]. These arrays were used to determine the event time and to iden- 


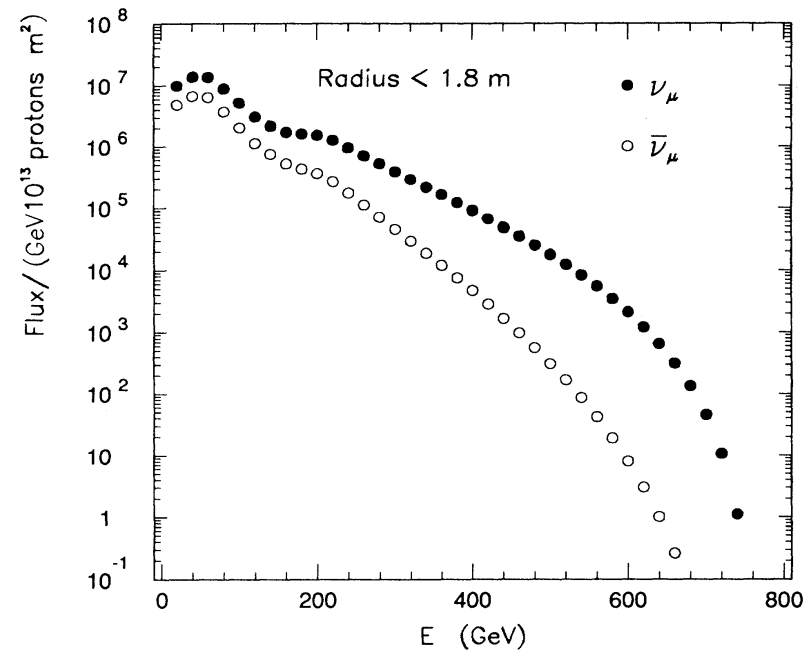

FIG. 2. Calculated $\nu_{\mu}$ and $\bar{\nu}_{\mu}$ beam energy spectra at the 15-foot bubble chamber for neutrinos with a perpendicular distance from the beam axis smaller than $1.8 \mathrm{~m}$.

tify muons. The IPF consisted of a double layer of vertical proportional tubes located around the bubble chamber inner vessel. The IPF was divided into two sections with a total coverage of $270^{\circ}$ in azimuth. The EMI consisted of three vertical planes (EMIA, EMIB, and EMIC) of proportional tubes, located downstream from the bubble chamber. [The smallest (EMIA) plane was to one side and was not needed in this analysis.] The EMIB plane was separated from the bubble chamber liquid by 3 to 5 hadronic interaction lengths of material. An additional 4 to 6 hadronic interaction lengths of material separated the EMIC plane from the EMIB plane. The EMIB plane contained three double layers of tubes to measure the position along the horizontal and vertical directions, as well

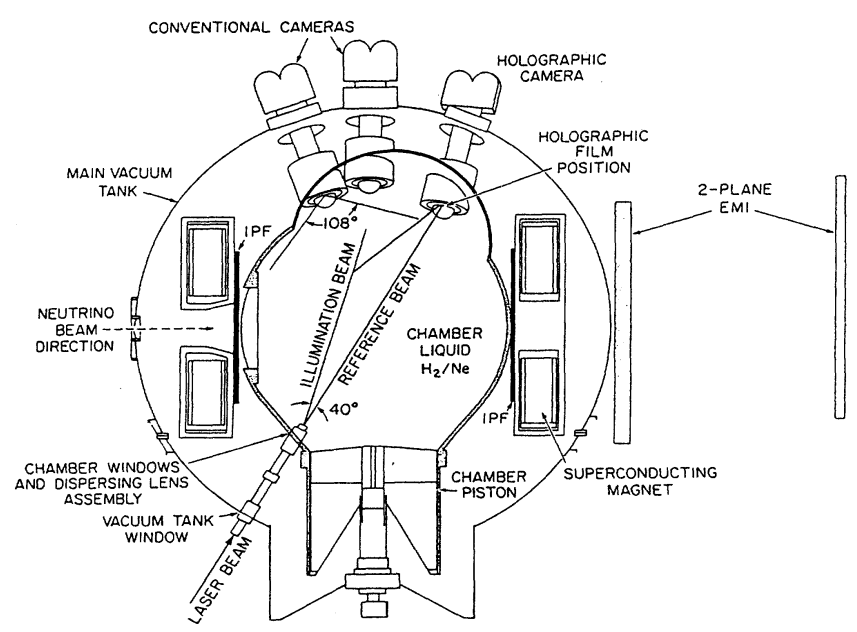

FIG. 3. Side view of the 15 -foot bubble chamber. as along a direction $63.5^{\circ}$ from the vertical. The EMIC plane contained only two double layers measuring the position along the horizontal and vertical directions. Both EMIB and EMIC planes covered an area of $668 \times 363$ $\mathrm{cm}^{2}$.

For this study, 335000 frames of film were analyzed, corresponding to a total of $5.5 \times 10^{17}$ protons on target. We measured all two-prong interactions in this sample as well as all four-prong interactions in a subsample corresponding to $4.2 \times 10^{17}$ protons on target. The measurements included the associated neutral strange particles and $\gamma$ conversions originating from the primary interaction. After measurement, any $\gamma$ conversions that were inconsistent with coming from the primary interaction or that were assigned as coming from external bremsstrahlung processes were discarded. Events of all multiplicities were also measured on $10 \%$ of the exposure to provide an inclusive sample of charged-current interactions for normalization. From this subsample, the total number of (anti)neutrino-neon charged-current events (with $p_{\mu}>10 \mathrm{GeV} / c$ ) is estimated to be 28000 .

The events are required to lie inside a restricted fiducial volume $\left(\sim 10 \mathrm{~m}^{3}\right)$ where the visibility is good, to ensure good particle detection and measurement accuracy. This volume is smaller than that used in our previous analyses $[10,24]$.

Events are required to have at least one identified muon with momentum greater than $10 \mathrm{GeV} / c$. (Note that this implies a cut on the total event energy $E>10 \mathrm{GeV}$.) Muon tracks leave the bubble chamber without interacting and are required to have good matches in at least two of the three EMIB coordinates and in both EMIC coordinates. These identification criteria were found to yield good muon identification efficiency $(\sim 94 \%)$ with little background $(\lesssim 0.5 \%)$ from hadron punchthrough, $\pi / K$ decays and/or accidental associations.

With the exception of low momentum protons $(p \leq 800$ $\mathrm{MeV} / c)$, most charged hadrons cannot be identified and are subsequently treated as pions. Here, all heavily ionizing short straight tracks together with the identified protons with momentum smaller than $800 \mathrm{MeV} / c$ are referred to as "stubs"; many are nuclear fragments resulting from the breakup of the neon nucleus. These stubs are not counted as prongs and they are not used in the computation of the kinematical variables characterizing the events.

To study reactions (1) and (2), we select events with two oppositely charged particles, compatible with the final state $\mu^{ \pm} \pi^{\mp}$ or $\mu^{ \pm} \pi^{\mp} \gamma \gamma$, that are not associated with any upstream interaction. These events may or may not contain additional stubs. Furthermore, the events are required to have definite final state charge (no track with $\frac{\Delta p}{p}>60 \%$ ).

In this analysis, we have used the Monte Carlo simulation of the coherent production of $\pi$ and $\rho$ mesons described in the Appendix. The simulation incorporates experimental conditions such as the (anti)neutrino energy spectra and the smearing effects due to measurement uncertainties and due to the uncertainty in the beam direction [23]. 


\section{SINGLE PION PRODUCTION}

\section{A. Extraction of the signal}

To extract the coherent signal, we use the characteristic exponential decrease of the coherent cross section in the variable $|t|$, the square of the four-momentum transfer to the nucleus. The value of $|t|$ can be calculated even when the momentum transferred to the nucleus is very small and the recoil nucleus remains undetected. In this case, $|t|$ can be computed to a good approximation by using the four-momenta of the observed final state particles:

$$
|t| \simeq\left(\sum_{i}\left(E_{i}-p_{i L}\right)\right)^{2}+\left(\sum_{i} \mathbf{p}_{i T}\right)^{2},
$$

where $E_{i}$ is the energy and $p_{i L}\left(\mathbf{p}_{i T}\right)$ is the longitudinal (transverse) momentum of particle $i$, relative to the neutrino direction; the sum extends over all observed particles except stubs. In the case of incoherent interactions, the quantity $|t|$ defined in Eq. (3) remains useful for separation of coherent and incoherent processes.

To extract the signal we follow the method used in most previous experiments and described in Ref. [25]. The events are separated into two categories: events with or without stubs. The events with stubs are necessarily incoherent since the nucleus broke up, whereas the events without stubs are a mixture of coherent and incoherent events. Thus, the distributions for events with stubs may be used to estimate the incoherent background affecting the distributions for events without stubs.

In order to study reactions (1), we select events with two prongs and no primary neutral strange particles or $\gamma$ conversions. We find $136 \mu^{-} \pi^{+}\left(34 \mu^{+} \pi^{-}\right)$events without stubs and $60 \mu^{-} \pi^{+}\left(20 \mu^{+} \pi^{-}\right)$events with stubs. The $|t|$ distribution for events without stubs is shown in Fig. 4, together with the distribution for events with stubs (dashed) normalized to the former distribution at $|t|>0.2 \mathrm{GeV}^{2}$. A large peak is observed in the distribution for events without stubs at $|t|<0.1 \mathrm{GeV}^{2}$ : there are 64 such events whereas there are only 7 events with stubs in the same range of $|t|$. After subtraction of the normalized background, the signal is estimated to be $(53.3 \pm 9.4)$ events at $|t|<0.1 \mathrm{GeV}^{2}$. Table I presents the number of events found in the $\nu_{\mu}$ and $\bar{\nu}_{\mu}$ channels separately and

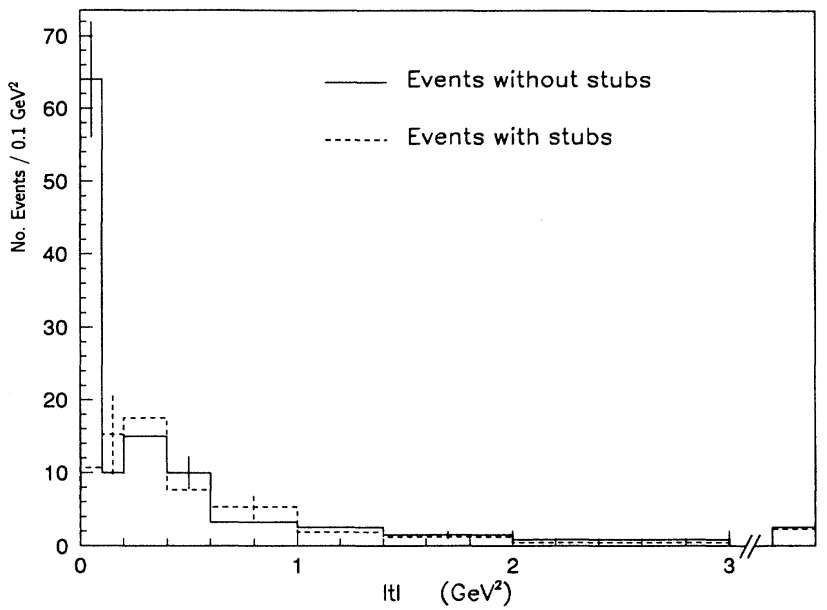

FIG. 4. $|t|$ distributions for $\mu^{ \pm} \pi^{\mp}$ events, normalized at $|t|>0.2 \mathrm{GeV}^{2}$.

combined. It should be noted that the magnitude of the signal is not significantly affected by our particular choice of background sample. For example, reducing the maximum stub momentum from 800 to $350 \mathrm{MeV} / c$ or selecting only events with one or with more than one stub, does not significantly change the size of the signal.

The background due to coherent $\rho$ production where both $\gamma$ conversions are undetected is estimated to be less than 0.5 events at $|t|<0.1 \mathrm{GeV}^{2}$, and that due to coherent $a_{1}$ production (with undetected $\gamma$ conversions) is less than 0.1 events [23]. Therefore, these background sources are neglected.

\section{B. Corrections to the coherent signal}

The observed signal is corrected for the following cuts and losses.

(i) The correction for events removed by the $\frac{\Delta p}{p}<60 \%$ cut was estimated to be $(1.103 \pm 0.011)$.

(ii) The scanning efficiency for $\mu \pi$ events was assessed to be $(72.9 \pm 6.5) \%$ from the results of a double scan and a special scan in which the IPF and EMI data were used to select frames with a muon candidate originating in the

TABLE I. Single pion coherent signal and cross section.

\begin{tabular}{lccc}
\hline \hline & $\nu$ & $\bar{\nu}$ & All \\
\hline Events without stubs & 136 & 34 & 170 \\
Events with stubs & 60 & 20 & 80 \\
Events without stubs $|t|<0.1 \mathrm{GeV}^{2}$ & 47 & 17 & 64 \\
Events with stubs $|t|<0.1 \mathrm{GeV}^{2}$ & 4 & 3 & 7 \\
Signal $|t|<0.1 \mathrm{GeV}$ (observed) & $40.5 \pm 8.1$ & $13.6 \pm 5.0$ & $53.3 \pm 9.4$ \\
\hline Signal (corrected) & $69.7 \pm 15.9$ & $22.8 \pm 8.7$ & $91.1 \pm 18.7$ \\
$N_{\mathrm{CC}}$ & $24197 \pm 609$ & $4011 \pm 308$ & $28208 \pm 682$ \\
$N_{\text {coh }} / N_{\mathrm{CC}}\left(\times 10^{-2}\right)$ & $0.29 \pm 0.07$ & $0.57 \pm 0.22$ & $0.32 \pm 0.07$ \\
$\sigma_{\text {coh }}\left(\times 10^{-38} \mathrm{~cm}^{2}\right)$ & $3.5 \pm 0.8$ & $2.7 \pm 1.1$ & $3.2 \pm 0.7$ \\
\hline \hline
\end{tabular}


bubble chamber.

(iii) The fraction of events with $E>10 \mathrm{GeV}$ passing the muon momentum cut $p_{\mu}>10 \mathrm{GeV} / c$ was determined to be $(98.7 \pm 0.1) \%$ using the coherent Monte Carlo simulation.

(iv) The fraction of events passing the $|t|<0.1 \mathrm{GeV}^{2}$ cut was estimated using the simulation, and found to be $(89.8 \pm 5.2) \%$, where the error includes the systematic error due to the uncertainty in the beam direction.

The overall correction factor is thus $(1.71 \pm 0.18)$ for $\left(\nu_{\mu}+\bar{\nu}_{\mu}\right)$ interactions combined. (See Table II for the correction factors for $\nu_{\mu}$, and $\bar{\nu}_{\mu}$ interactions separately.) As a result, the corrected signal becomes $(69.7 \pm 15.9)$ for $\nu_{\mu},(22.8 \pm 8.7)$ for $\bar{\nu}_{\mu}$, and $(91.1 \pm 18.7)$ for $\left(\nu_{\mu}+\right.$ $\left.\bar{\nu}_{\mu}\right)$ interactions.

\section{Cross-section results}

Using our inclusive sample of charged-current events, the total number of charged-current interactions on neon corresponding to our two-prong sample was estimated to be $(28208 \pm 682)$, containing (24197 \pm 609$) \nu_{\mu}$ and $(4011 \pm 308) \bar{\nu}_{\mu}$ interactions. These numbers take into account the scanning losses, the loss of events too complex to be measured, the fraction of events on hydrogen, and the loss of events with $E>10 \mathrm{GeV}$ which have $p_{\mu}<10$ $\mathrm{GeV} / c$. The total correction factor was estimated to be $(1.111 \pm 0.004)$ for $\nu_{\mu}$ events and $(1.050 \pm 0.052)$ for $\bar{\nu}_{\mu}$ events. The coherent pion production rates are thus found to be $(0.29 \pm 0.07) \%$ in the case of $\nu_{\mu}$ interactions, $(0.57 \pm 0.22) \%$ in the case of $\bar{\nu}_{\mu}$ interactions, and $(0.32 \pm 0.07) \%$ for $\left(\nu_{\mu}+\bar{\nu}_{\mu}\right)$ interactions combined. These rates are averaged over our (anti)neutrino energy spectra for $E>10 \mathrm{GeV}$. A higher rate in $\bar{\nu}_{\mu}$ interactions is expected because the total cross section for $\bar{\nu}_{\mu}$ chargedcurrent interactions is about half that for $\nu_{\mu}$ chargedcurrent interactions whereas the coherent cross section remains essentially the same for $\nu_{\mu}$ and $\bar{\nu}_{\mu}$.

The corresponding coherent cross sections are computed according to $\sigma_{\mathrm{coh}}=\frac{N_{\mathrm{coh}}}{N_{\mathrm{CC}}} A \sigma_{\mathrm{CC}}$, where $N_{\mathrm{coh}}$ and $N_{\mathrm{CC}}$ are the corrected numbers of coherent and chargedcurrent interactions on neon, and $A=20$ for neon. The total charged-current cross section per nucleon $\sigma_{\mathrm{CC}}$ is evaluated using the cross-section measurements from Ref. [26] and the computed average $\nu_{\mu}$ and $\bar{\nu}_{\mu}$ beam energies for $E>10 \mathrm{GeV}:\left\langle E_{\nu}\right\rangle=91.1 \mathrm{GeV}$ and $\left\langle E_{\bar{\nu}}\right\rangle=74.5$ $\mathrm{GeV}$. This gives an average beam energy of $86.3 \mathrm{GeV}$ for $\left(\nu_{\mu}+\bar{\nu}_{\mu}\right)$ combined. As a result, the cross sections for reactions (1) are found to be

$$
\begin{aligned}
& \sigma_{\mathrm{coh}}\left(\nu_{\mu} \mathrm{Ne} \rightarrow \mu^{-} \pi^{+} \mathrm{Ne}\right) \\
& \quad=(3.5 \pm 0.8) \times 10^{-38} \mathrm{~cm}^{2} / \text { neon nucleus } \\
& \sigma_{\mathrm{coh}}\left(\bar{\nu}_{\mu} \mathrm{Ne} \rightarrow \mu^{+} \pi^{-} \mathrm{Ne}\right) \\
& \quad=(2.7 \pm 1.1) \times 10^{-38} \mathrm{~cm}^{2} / \text { neon nucleus }
\end{aligned}
$$

and for the combined $\left(\nu_{\mu}+\bar{\nu}_{\mu}\right)$ data

$$
\begin{aligned}
\sigma_{\mathrm{coh}}\left[\left(\nu_{\mu}+\bar{\nu}_{\mu}\right) \mathrm{Ne} \rightarrow \mu \pi \mathrm{Ne}\right] \\
=(3.2 \pm 0.7) \times 10^{-38} \mathrm{~cm}^{2} / \text { neon nucleus }
\end{aligned}
$$

averaged over our (anti)neutrino energy spectra $(E>$ $10 \mathrm{GeV}$ ). The cross section (4) agrees with the predicted cross section: we computed a value of $2.8 \times 10^{-38}$ $\mathrm{cm}^{2} /$ neon nucleus using the $\rho \pi$ cut form factor [see Eq. (A3)] and $2.5 \times 10^{-38} \mathrm{~cm}^{2} /$ neon nucleus using the $a_{1}$ pole form factor [see Eq. (A2)]. As for the number of observed events with $|t|<0.1 \mathrm{GeV}^{2}$ and $p_{\mu}>10 \mathrm{GeV} / c$, the signal of $(53 \pm 9)$ events is to be compared with predicted numbers of $(46 \pm 5)$ events for the $\rho \pi$ cut form factor and $(41 \pm 4)$ events for the $a_{1}$ pole form factor (the errors correspond to the uncertainty in the correction factor; see Table II).

In order to compare these results with the most significant measurements from previous experiments, we have computed the combined $\left(\nu_{\mu}+\bar{\nu}_{\mu}\right)$ coherent cross section for energies below and above $80 \mathrm{GeV}$. Figure 5 shows the cross section as a function of energy along with the predictions of the model for different parametrizations of the pion-nucleus cross section and form factors. It is found that the measured cross sections are in reasonable agreement with the predictions and that the BelkovKopeliovich approximation [see Eq. (A6)] predicts cross sections which are $\sim 15 \%$ higher than those obtained with our full Glauber model simulation [see Eqs. (A4) and (A5)].

The above analysis was repeated for events with $\nu=$ $E-E_{\mu}>2 \mathrm{GeV}$. Such a cut is applied to avoid the kinematical region where low mass nucleon resonance production dominates the neutrino and pion cross sections and to select a regime where coherent interactions are more prominent (i.e., the region with low $Q^{2}$ and high $\nu$ ). With that additional cut, we find $66 \mu^{-} \pi^{+}\left(17 \mu^{+} \pi^{-}\right)$events

TABLE II. Correction factors for coherent single pion events.

\begin{tabular}{lccc}
\hline \hline & $\nu$ & $\bar{\nu}$ & All \\
\hline$\frac{\Delta p}{p}$ cut correction $\left(c_{1}\right)$ & $1.104 \pm 0.011$ & $1.104 \pm 0.011$ & $1.104 \pm 0.011$ \\
Scanning efficiency $\left(c_{2}\right)$ & $0.729 \pm 0.065$ & $0.729 \pm 0.065$ & $0.729 \pm 0.065$ \\
Fraction with $p_{\mu}>10 \mathrm{GeV} / c\left(c_{3}\right)$ & $0.988 \pm 0.001$ & $0.984 \pm 0.001$ & $0.987 \pm 0.001$ \\
Fraction with $|t|<0.1 \mathrm{GeV}^{2}\left(c_{4}\right)$ & $0.890 \pm 0.058$ & $0.916 \pm 0.042$ & $0.898 \pm 0.052$ \\
\hline $\begin{array}{l}\text { Total correction factor } \\
\quad c_{1} /\left(c_{2} c_{3} c_{4}\right)\end{array}$ & $1.72 \pm 0.19$ & $1.68 \pm 0.17$ & $1.71 \pm 0.18$ \\
\hline \hline
\end{tabular}




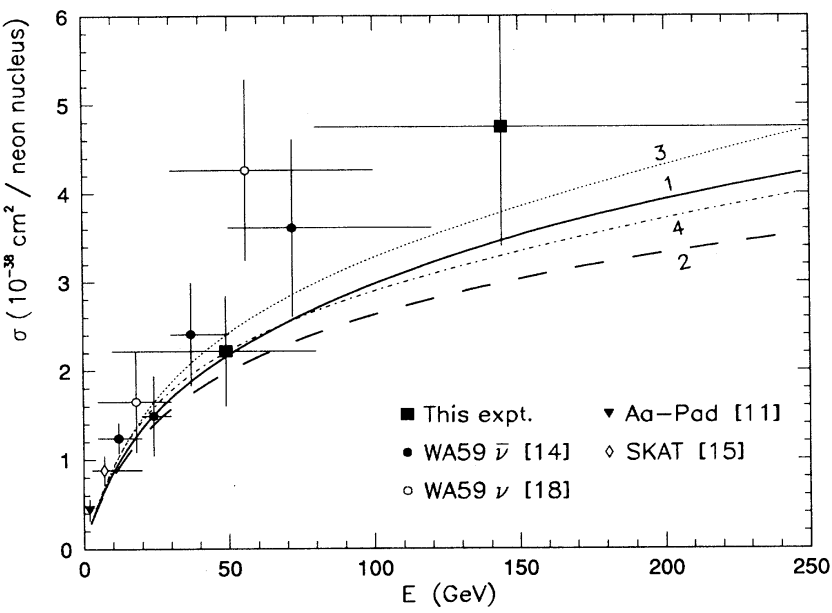

FIG. 5. Single pion coherent cross section as a function of energy. The data points correspond to the combined $\left(\nu_{\mu}\right.$ $\left.+\bar{\nu}_{\mu}\right)$ cross sections except for the WA59 data. The curves represent the predictions of the model (1: Glauber $\rho \pi$ cut, 2: Glauber $a_{1}$ pole, 3: Belkov-Kopeliovich $\rho \pi$ cut, 4: BelkovKopeliovich $a_{1}$ pole). Results from other experiments have been scaled to correspond to charged-current interactions on neon nuclei, where necessary.

without stubs and $15 \mu^{-} \pi^{+}\left(3 \mu^{+} \pi^{-}\right)$events with stubs. At $|t|<0.1 \mathrm{GeV}^{2}$, there are (is) 46 (1) event(s) without (with) stubs. The uncorrected signal is thus found to be $(43.6 \pm 7.7)$ events, yielding a corrected signal of $(75.0 \pm 15.4)$ events with $\nu>2 \mathrm{GeV}$ and a corresponding production rate of $(0.27 \pm 0.06) \%$ for $\left(\nu_{\mu}+\bar{\nu}_{\mu}\right)$ combined. The resulting cross section is determined to be
$(2.7 \pm 0.6) \times 10^{-38} \mathrm{~cm}^{2} /$ neon nucleus. This measurement is in agreement with the predicted cross section of $2.4(2.1) \times 10^{-38} \mathrm{~cm}^{2} /$ neon nucleus for the Regge $\rho \pi$ cut $\left(a_{1}\right.$ pole) form factor, computed using the Glauber model relations.

\section{Kinematical characteristics}

The kinematical properties of the coherent $\mu^{-} \pi^{+}$and $\mu^{+} \pi^{-}$events at $|t|<0.1 \mathrm{GeV}^{2}$ and $\nu>2 \mathrm{GeV}$ were examined in terms of the following kinematical variables: the total energy of the event $E$, the energy transfer $\nu=E-E_{\mu}$, the negative of the square of the fourmomentum transfer from the leptons to the hadrons $Q^{2}=2 E\left(E-p_{\mu L}\right)-m_{\mu}^{2}$, the invariant mass of the hadronic system $W=\left(m_{N}^{2}+2 m_{N} \nu-Q^{2}\right)^{1 / 2}\left(m_{N}\right.$ is the nucleon mass $)$, the Bjorken variables $x=Q^{2} /\left(2 m_{N} \nu\right)$ and $y=\nu / E$, and the momentum transfer variables $|t|, t_{\min }$, the minimum value of $|t|$ required to produce a final state of effective mass $m$ [ $t_{\min }$ is approximately $\left.\left(\frac{Q^{2}+m^{2}}{2 \nu}\right)^{2}\right]$, and $t^{\prime}=|t|-t_{\min }$. The distributions of the variables $E, \nu, Q^{2}, W, x$ and $y$ are shown in Fig. 6. The distributions of the variables $|t|, t_{\min }$, and $t^{\prime}$ are shown in Fig. 7. Also shown are the predictions of the model, computed using the $\rho \pi$ cut form factor and our full Glauber model simulation [see Eqs. (A4) and (A5)]. These predictions also include the experimental smearing effects. Table III compares the average values of these variables with the predictions of the model [27] for the $\rho \pi$ cut form factor, as well as for the propagator form factor with the axial mass taken as the $a_{1}$ mass ( $a_{1}$ pole) or according to the spectral density function distribution measured by the ARGUS Collaboration in a study of $\tau \rightarrow a_{1} \nu_{\tau}$ decays $[28]$.
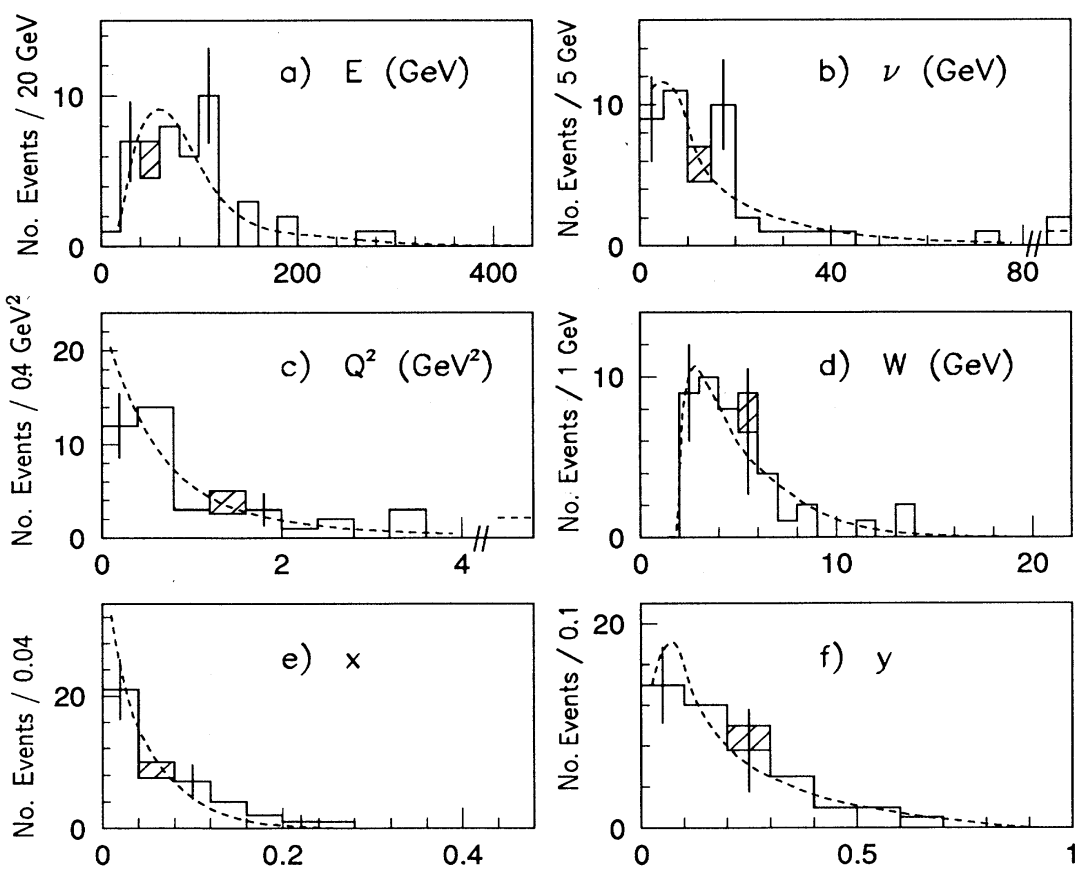

FIG. 6. Kinematical variables distributions for $\mu^{ \pm} \pi^{\mp}$ events at $|t|<0.1 \mathrm{GeV}^{2}$ and $\nu>2 \mathrm{GeV}$ : (a) $E$, (b) $\nu$, (c) $Q^{2}$, (d) $W$, (e) $x$, (f) $y$. The curves represent the predictions of the model normalized to the signal. The one event with stubs (shown cross-hatched) has been normalized and subtracted. 
TABLE III. Average values of kinematical variables for single pion coherent events at $|t|<0.1$ $\mathrm{GeV}^{2}$ and $\nu>2 \mathrm{GeV}$. The predictions of the model are given for different form factor parametrizations.

\begin{tabular}{lcccc}
\hline \hline & Data & & Model & \\
& & $\rho \pi$ cut & $a_{1}$ pole & $\begin{array}{c}\text { Spectral } \\
\text { function }\end{array}$ \\
\hline$\langle E\rangle(\mathrm{GeV})$ & $92.6 \pm 8.5$ & 94.3 & 93.3 & 93.7 \\
$\langle\nu\rangle(\mathrm{GeV})$ & $17.8 \pm 2.5$ & 17.5 & 16.3 & 16.4 \\
$\left\langle Q^{2}\right\rangle\left(\mathrm{GeV}^{2}\right)$ & $1.36 \pm 0.23$ & 1.09 & 0.89 & 0.89 \\
$\langle W\rangle\left(\mathrm{GeV}^{2}\right.$ & $5.08 \pm 0.38$ & 5.09 & 4.92 & 4.94 \\
$\langle x\rangle$ & $0.065 \pm 0.009$ & 0.044 & 0.041 & 0.041 \\
$\langle y\rangle$ & $0.202 \pm 0.022$ & 0.205 & 0.194 & 0.194 \\
$\langle|t|\rangle\left(\mathrm{GeV}^{2}\right)$ & $0.034 \pm 0.003$ & 0.025 & 0.025 & 0.024 \\
$\left\langle t_{\min }\right\rangle\left(\mathrm{GeV}^{2}\right)$ & $0.007 \pm 0.002$ & 0.003 & 0.003 & 0.003 \\
$\left\langle t^{\prime}\right\rangle\left(\mathrm{GeV}^{2}\right)$ & $0.027 \pm 0.003$ & 0.022 & 0.022 & 0.021 \\
$t^{\prime}$ slope $\left(\mathrm{GeV}^{-2}\right)$ & $30 \pm 7$ & 43 & 44 & 44 \\
\hline \hline
\end{tabular}

As seen in Table III, there is good overall agreement between the data and the model predictions. The kinematical characteristics and the measured cross sections (Fig. 5) appear to slightly favor the $\rho \pi$ cut form factor over the $a_{1}$ pole or spectral function forms. The $|t|$ and $t^{\prime}$ distributions appear to be somewhat flatter than predicted. The $t^{\prime}$ distribution was parametrized as an exponential in accordance with Eq. (A6) and the slope, extracted using the maximum likelihood method, was found to be $(30 \pm 7) \mathrm{GeV}^{-2}$. This value is slightly smaller than predicted by the model (see Table III), yet it is consistent with the interpretation of the signal at $|t|<0.1$ $\mathrm{GeV}^{2}$ as due to coherent interactions on neon nuclei. Indeed, a slope of 6-9 $\mathrm{GeV}^{-2}$ [29] might be expected if those events were due to diffractive pion production on single nucleons.

Note that the kinematical characteristics obtained using the Belkov and Kopeliovich approximation are very similar to those obtained using the full Glauber model.

\section{SINGLE $\rho$ MESON PRODUCTION}

\section{A. Extraction of the signal}

In order to study reactions (2), we select events with two prongs and two associated $\gamma$ conversions. We find
$149 \mu^{-} \pi^{+} \gamma \gamma$ and $30 \mu^{+} \pi^{-} \gamma \gamma$ events (with or without stubs) satisfying our selection criteria. Figure 8 shows the $\gamma \gamma$ invariant mass distribution for these events. A clear peak at the $\pi^{0}$ mass is observed. The $\gamma \gamma$ pairs are then constrained to fit the decay $\pi^{0} \rightarrow \gamma \gamma$. The $\pi^{0}$ fit probability distribution (Fig. 9) is consistent with a uniform distribution, and we require this probability to be greater than 0.01 for the fit to be retained. As a result, $105 \mu^{-} \pi^{+} \pi^{0}$ and $21 \mu^{+} \pi^{-} \pi^{0}$ events are selected.

We follow the procedure outlined in Sec. III A to extract the coherent signal. The $|t|$ distributions for the $70 \mu^{-} \pi^{+} \pi^{0}+14 \mu^{+} \pi^{-} \pi^{0}$ events without stubs and 35 $\mu^{-} \pi^{+} \pi^{0}+7 \mu^{+} \pi^{-} \pi^{0}$ events with stubs are shown in Fig. 10. The distribution for events with stubs is normalized to that for events without stubs at $|t|>0.2$ $\mathrm{GeV}^{2}$. As in the case of $\mu \pi$ events, we observe a large excess of events without stubs at $|t|<0.1 \mathrm{GeV}^{2}$ : there are 26 such events and only 4 events with stubs. After proper normalization and subtraction of the background, the coherent signal at $|t|<0.1 \mathrm{GeV}^{2}$ is determined to be $(19.2 \pm 6.8)$ events. Among the events at $|t|<0.1 \mathrm{GeV}^{2}$, there are 22 (3) $\mu^{-} \pi^{+} \pi^{0}$ and 4 (1) $\mu^{+} \pi^{-} \pi^{0}$ events without (with) stubs. These results are summarized in Table IV.

Figure 11 shows the $\pi^{\mp} \pi^{0}$ invariant mass distribution for $\mu^{ \pm} \pi^{\mp} \pi^{0}$ events at $|t|<0.1 \mathrm{GeV}^{2}$. Also shown is the
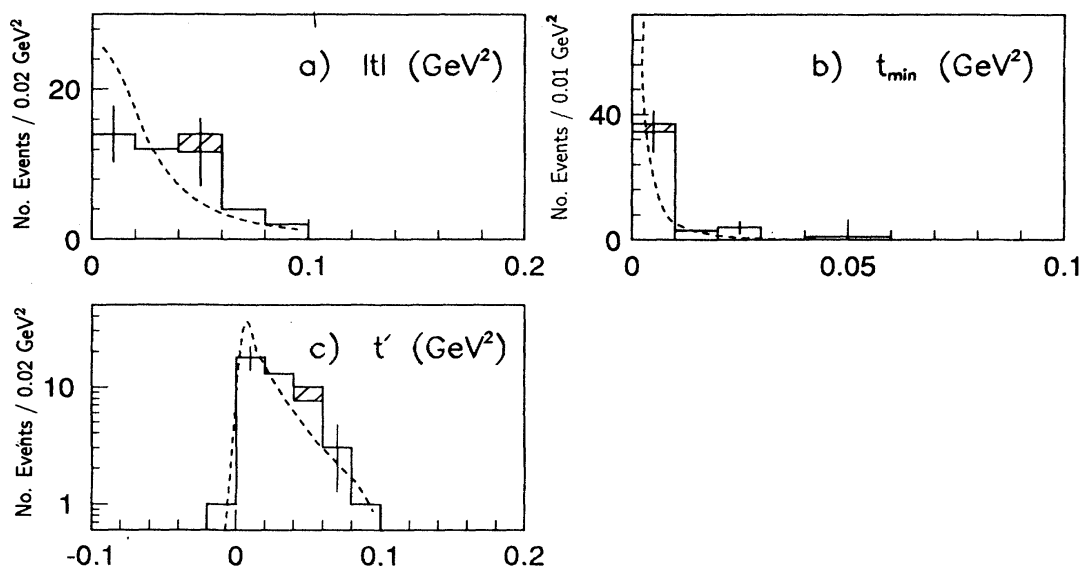

FIG. 7. Distributions of momentum transfer variables for $\mu^{ \pm} \pi^{\mp}$ events at $|t|<$ $0.1 \mathrm{GeV}^{2}$ and $\nu>2 \mathrm{GeV}$ : (a) $|t|$, (b) $t_{\min }$, (c) $t^{\prime}$. The curves represent the predictions of the model normalized to the signal. The one event with stubs (shown cross-hatched) has been normalized and subtracted. 


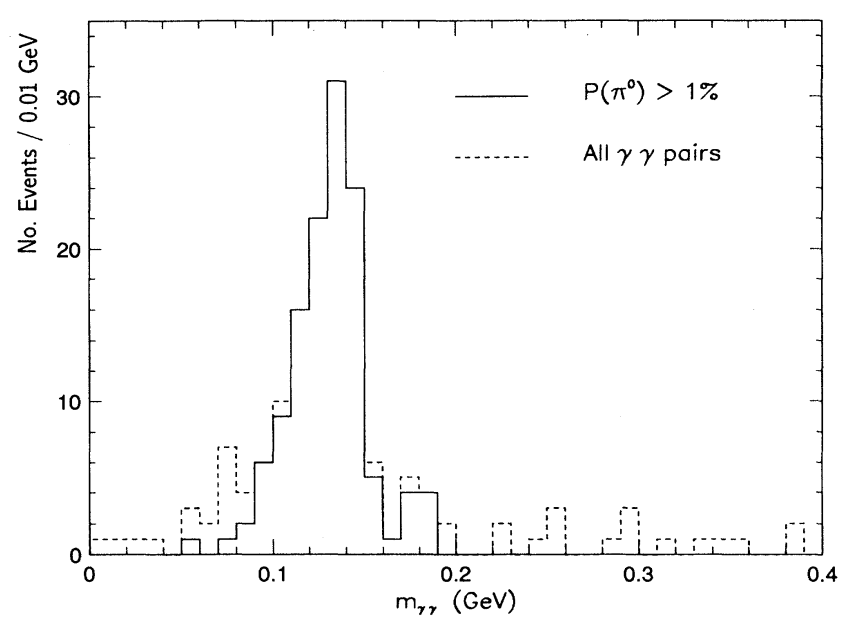

FIG. 8. Invariant $\gamma \gamma$ mass distribution for the $\mu^{ \pm} \pi^{\mp} \gamma \gamma$ events with $\pi^{0}$ fit probability greater than $1 \%$; the dashed histogram, including 14 events with $m_{\gamma \gamma}>0.4 \mathrm{GeV}$, is for pairs with no probability cut.

TABLE IV. Single $\rho$ coherent signal and cross section.

\begin{tabular}{lc}
\hline \hline & All \\
\hline Events without stubs & 84 \\
Events with stubs & 42 \\
Events without stubs $|t|<0.1 \mathrm{GeV}^{2}$ & 26 \\
Events with stubs $|t|<0.1 \mathrm{GeV}^{2}$ & 4 \\
Signal $|t|<0.1 \mathrm{GeV}$ (observed) & $19.2 \pm 6.8$ \\
\hline Signal (corrected) & $59.7 \pm 23.1$ \\
$N_{\mathrm{CC}}$ & $28208 \pm 682$ \\
$N_{\text {coh }} / N_{\mathrm{CC}}\left(\times 10^{-2}\right)$ & $0.21 \pm 0.08$ \\
$\sigma_{\text {coh }}\left(\times 10^{-38} \mathrm{~cm}^{2}\right)$ & $2.1 \pm 0.8$ \\
\hline \hline
\end{tabular}

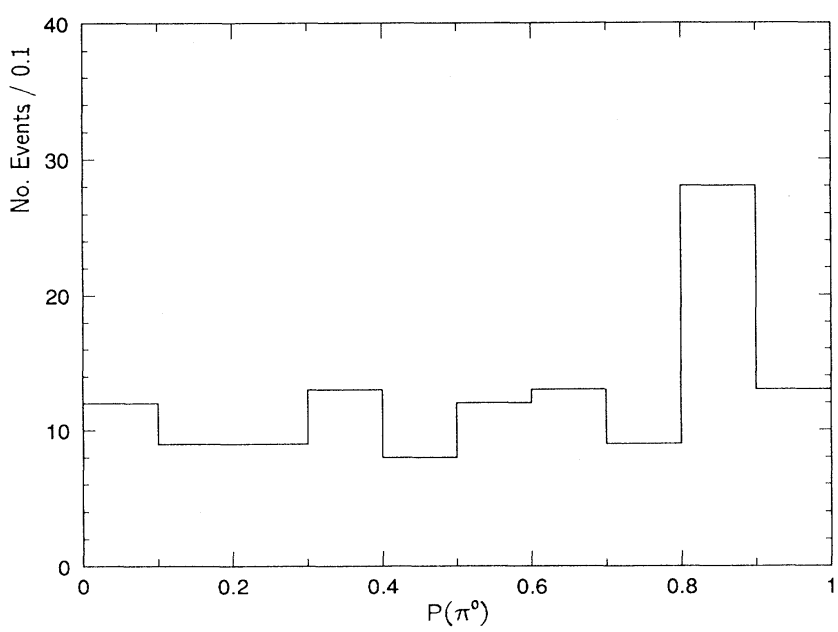

FIG. 9. $\pi^{0}$ fit probability distribution for $\gamma \gamma$ pairs with $P\left(\pi^{0}\right)>1 \%$.

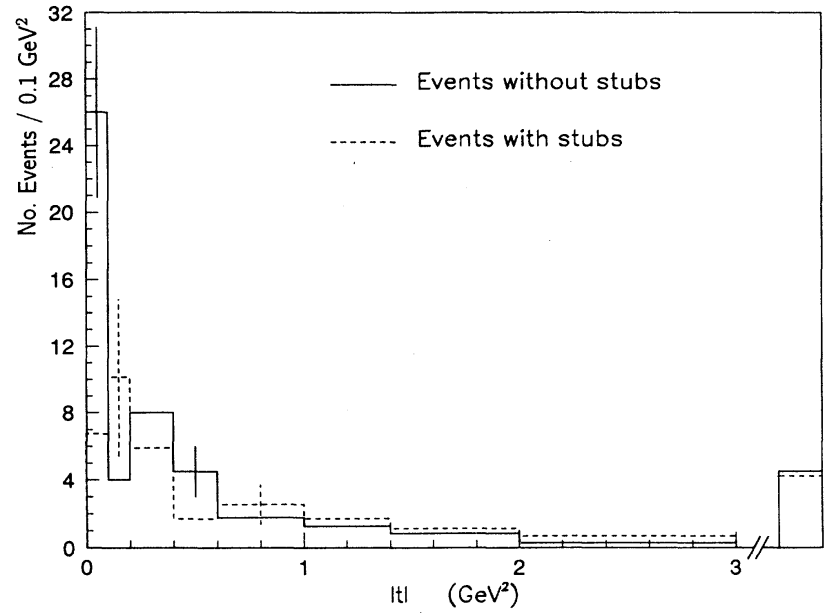

FIG. 10. $|t|$ distributions for $\mu^{ \pm} \pi^{\mp} \pi^{0}$ events, normalized at $|t|>0.2 \mathrm{GeV}^{2}$.

simulated $\rho$ line shape with or without a Ross-Stodolsky skewing factor $\left(m_{\rho} / m_{\pi \pi}\right)^{3}$ [30]. It is observed that the $\pi^{\mp} \pi^{0}$ pairs are compatible with originating from the decay of $\rho^{\mp}$ mesons.

To check the consistency of the analysis, we have also extracted the signal for $\mu^{ \pm} \pi^{\mp} \gamma$ events. An excess of $(9.3 \pm 6.0)$ events at $|t|<0.1 \mathrm{GeV}^{2}$ is found, in agreement with the expected $(7.2 \pm 2.6)$ events based on the observed $\mu^{ \pm} \pi^{\mp} \pi^{0}$ signal, the $\gamma$ detection efficiency, and the fraction of events surviving the $|t|<0.1 \mathrm{GeV}^{2}$ cut.

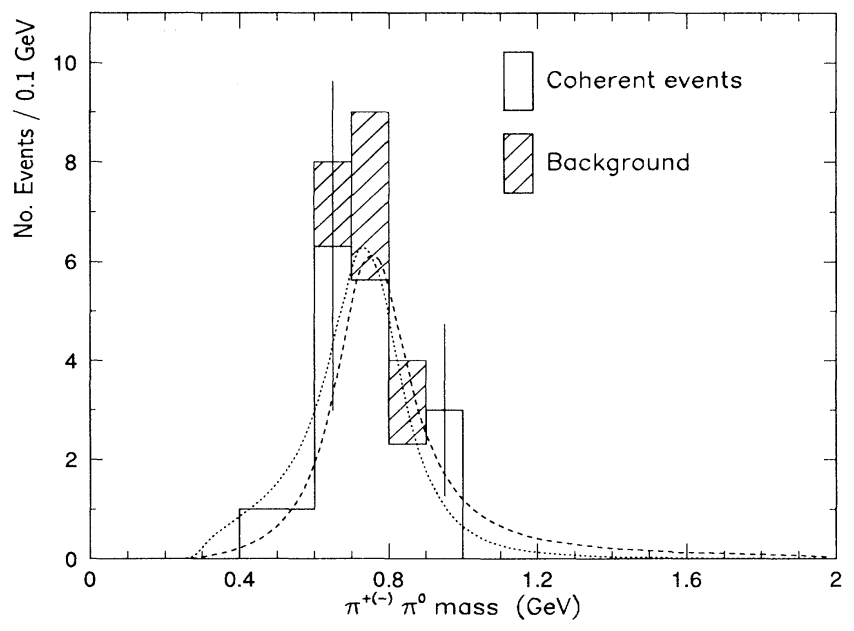

FIG. 11. $\pi^{\mp} \pi^{0}$ invariant mass for $\mu^{ \pm} \pi^{\mp} \pi^{0}$ events at $|t|<0.1 \mathrm{GeV}^{2}$. The events with stubs (shown cross-hatched) have been properly normalized and subtracted from the distribution for events without stubs. The dashed curve represents the simulated $\rho$ line shape normalized to the observed coherent signal. The dotted curve was obtained by applying a Ross-Stodolsky skewing factor $\left(m_{\rho} / m_{\pi \pi}\right)^{3}[30]$. 


\section{B. Corrections to the coherent signal}

The observed signal is corrected for the following cuts and losses.

(a) The correction for events removed by the $\frac{\Delta p}{p}<60 \%$ cut was estimated to be $(1.103 \pm 0.011)$.

(b) The scanning efficiency was estimated to be $(82.5 \pm 11.2) \%$ from the results of a double scan.

(c) The fraction of events with $E>10 \mathrm{GeV}$ passing the muon momentum cut $p_{\mu}>10 \mathrm{GeV} / c$ was found to be $(93.6 \pm 0.8) \%$ using the Monte Carlo simulation.

(d) The fraction of events passing the $|t|<0.1 \mathrm{GeV}^{2}$ cut computed using the Monte Carlo simulation was $(79.3 \pm 5.2) \%$, where the error includes the systematic error due to the uncertainty in the beam direction.

(e) The $\gamma$ detection efficiency was evaluated from the $\gamma$ multiplicity distribution for two-prong events and was found to be $(76.1 \pm 2.6) \%$.

The total correction factor is thus estimated to be $(3.11 \pm 0.49)$ (see Table V) and the corrected signal is $(59.7 \pm 23.1)$ events.

\section{Cross-section results}

The rate for coherent $\rho$ production is determined to be $(0.21 \pm 0.08) \%$ of the total number of charged-current events, averaged over our (anti)neutrino energy spectra. The combined $\left(\nu_{\mu}+\bar{\nu}_{\mu}\right)$ cross section is then computed as described in Sec. III C using the measured total charged-current cross section [26] and is found to be

$$
\begin{aligned}
\sigma_{\mathrm{coh}}\left[\left(\nu_{\mu}+\bar{\nu}_{\mu}\right) \mathrm{Ne} \rightarrow \mu \rho \mathrm{Ne}\right] \\
=(2.1 \pm 0.8) \times 10^{-38} \mathrm{~cm}^{2} / \text { neon nucleus }
\end{aligned}
$$

for an average $\left(\nu_{\mu}+\bar{\nu}_{\mu}\right)$ beam energy of $86.3 \mathrm{GeV}$ $(E>10 \mathrm{GeV})$. As pointed out in the Appendix, the predicted cross section is affected by the uncertainty in $R$, the ratio of longitudinal to transverse $\rho$ production. The predicted cross sections are $2.4 \times 10^{-38} \mathrm{~cm}^{2} /$ neon nucleus for $R=0$ and $3.8 \times 10^{-38} \mathrm{~cm}^{2} /$ neon nucleus for $R=0.4 Q^{2} / m_{\rho}^{2} \leq 1$. The corresponding numbers of observed events with $|t|<0.1 \mathrm{GeV}^{2}$ and $p_{\mu}>10 \mathrm{GeV} / c$ are thus predicted to be $(22 \pm 4)$ and $(34 \pm 5)$ events, respectively (the errors correspond to the uncertainty in the correction factor; see Table V), to be compared with the signal of $(19 \pm 7)$ events. Table IV summarizes the above results. The coherent $\rho$ production cross section as

TABLE V. Correction factors for coherent single $\rho$ events.

\begin{tabular}{lc}
\hline & All \\
\hline$\frac{\Delta p}{p}$ cut correction $\left(c_{1}\right)$ & $1.103 \pm 0.011$ \\
Scanning efficiency $\left(c_{2}\right)$ & $0.825 \pm 0.112$ \\
Fraction with $p_{\mu}>10 \mathrm{GeV} / c\left(c_{3}\right)$ & $0.936 \pm 0.008$ \\
Fraction with $|t|<0.1 \mathrm{GeV}^{2}\left(c_{4}\right)$ & $0.793 \pm 0.052$ \\
Gamma detection efficiency $\left(c_{5}\right)$ & $0.761 \pm 0.026$ \\
\hline Total correction factor & $3.11 \pm 0.49$ \\
$c_{1} /\left(c_{2} c_{3} c_{4} c_{5}^{2}\right)$ & 3.11 \\
\hline \hline
\end{tabular}

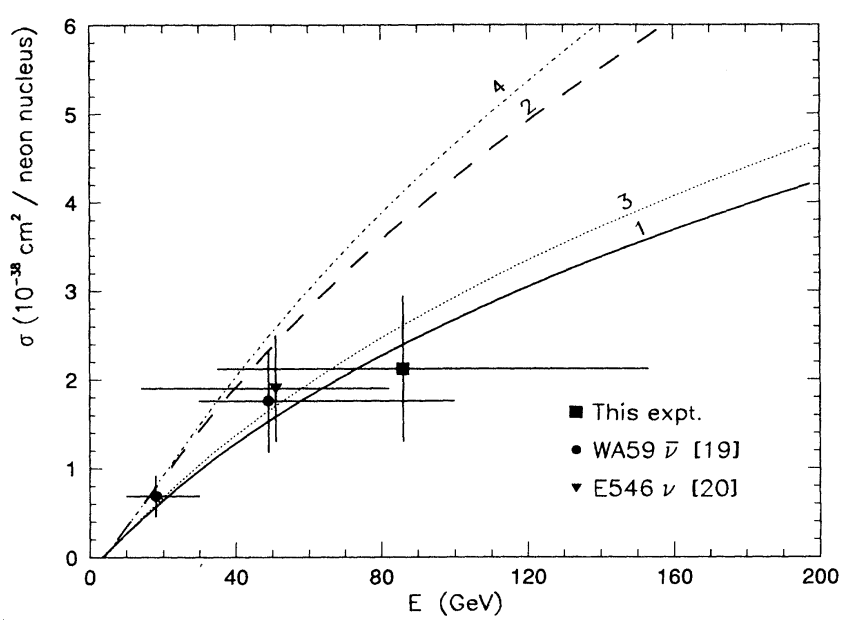

FIG. 12. Single $\rho$ coherent production cross section as a function of energy. For this experiment the combined $\left(\nu_{\mu}+\bar{\nu}_{\mu}\right)$ cross section is given. The curves represent the predictions of the model (1: Glauber $R=0,2$ : Glauber $R=0.4 Q^{2} / m_{\rho}^{2} \leq 1$, 3: Belkov-Kopeliovich $R=0$, 4: BelkovKopeliovich $\left.R=0.4 Q^{2} / m_{\rho}^{2} \leq 1\right)$. Note that, for the WA59 data, the horizontal error bar represents the range of energy covered, whereas for the data from this experiment and from E546 it represents the energy range containing $68 \%$ of the (anti)neutrino flux.

a function of energy is shown in Fig. 12 together with the WA59 [19] and E546 [20,31] results and the predictions of the model.

The angular characteristics of the events at $|t|<0.1$ $\mathrm{GeV}^{2}$ are used here to study the $\rho$ meson spin state. The cosine of the angle between the daughter charged pion and the flight direction of the parent $\rho$ meson in the $\rho$ center of mass $(\cos \theta)$ is shown in Fig. 13. The data

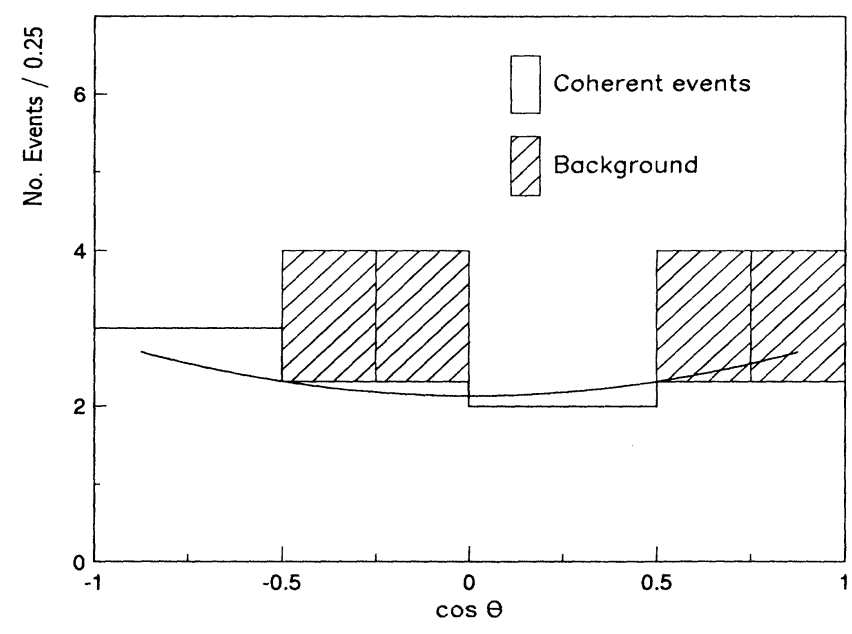

FIG. 13. Distribution of the cosine of the angle between the daughter charged pion and the parent $\rho$ flight direction in the $\rho$ center of mass. The curve represents the fit described in the text. 

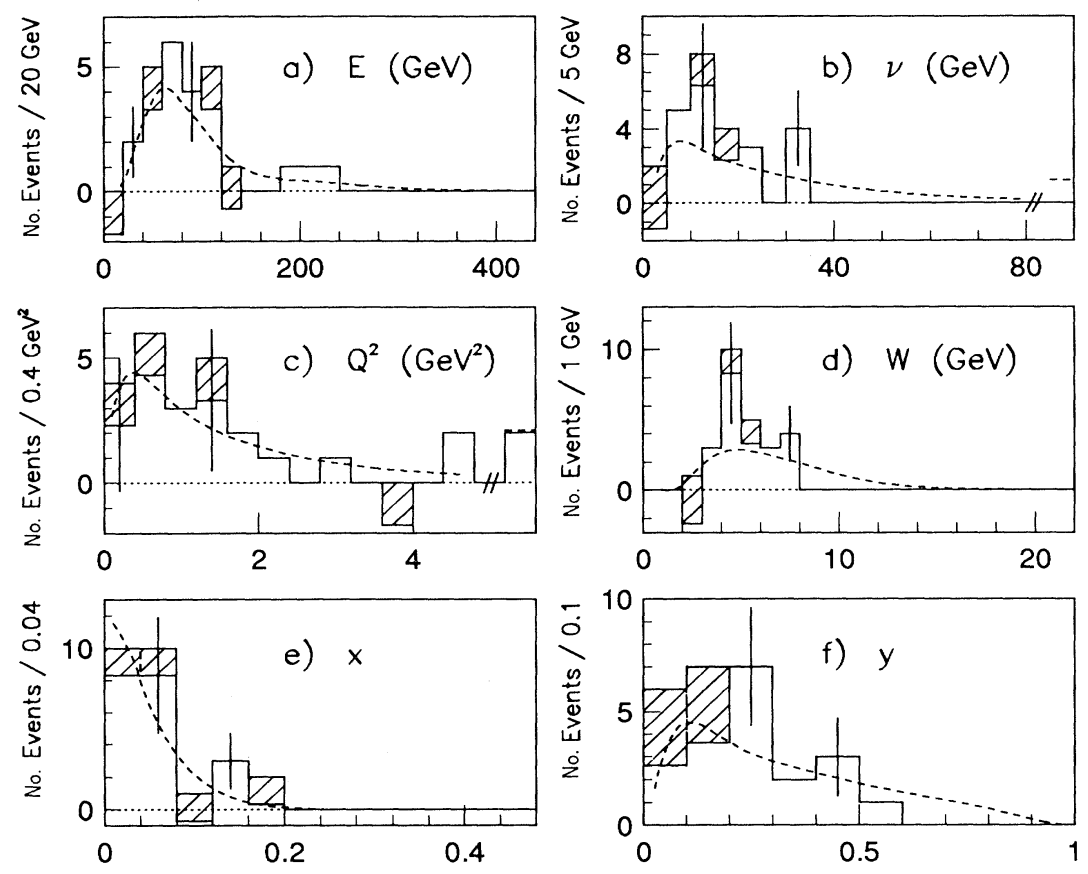

FIG. 14. Kinematical variables distributions for $\mu^{ \pm} \pi^{\mp} \pi^{0}$ events at $|t|<0.1 \mathrm{GeV}^{2}$ : (a) $E$, (b) $\nu$, (c) $Q^{2}$, (d) $W$, (e) $x$, (f) $y$. The curves represent the predictions of the model normalized to the signal. The events with stubs (shown cross-hatched) have been normalized and subtracted from the distributions for events without stubs. were fitted to the following $\rho$ decay angular distribution (derived assuming $s$-channel helicity conservation):

$$
W(\cos \theta)=\frac{3}{4}\left[1-r_{00}^{04}+\left(3 r_{00}^{04}-1\right) \cos ^{2} \theta\right],
$$

where $r_{00}^{04}$ is one of the $\rho^{ \pm}$density matrix elements [32] which represents the probability to produce a longitudinally polarized $\rho$ meson. As a result of the fit, the matrix element is determined to be $r_{00}^{04}=(0.40 \pm 0.18)$. Furthermore, the ratio of longitudinal and transverse production of $\rho$ mesons is related to $r_{00}^{04}$ via the expression

$$
R=\frac{\sigma_{L}}{\sigma_{T}}=\frac{1}{\epsilon} \frac{r_{00}^{04}}{1-r_{00}^{04}}
$$

where $\epsilon$ is defined according to Eq. (A1). Equation (6) implies, from the fitted value of $r_{00}^{04}$, a value $R=$ $(0.7 \pm 0.4)$, i.e., there is an indication of production of longitudinally polarized $\rho$ mesons. These results agree with those reported by the WA59 Collaboration [19]: $r_{00}^{04}=(0.41 \pm 0.19)$ which implies $R=(0.8 \pm 0.4)$. The E546 Collaboration finds $R \simeq 0.2$ from a simultaneous fit to the azimuthal and $\cos \theta$ decay distributions [20]. The parametrization $R=0.4 Q^{2} / m_{\rho}^{2} \leq 1$ gives an average value $\langle R\rangle=(0.69 \pm 0.08)$ for the events at $|t|<0.1 \mathrm{GeV}^{2}$. It should be pointed out, however, that our measured cross section seems to favor $R=0$ over $R=0.4 Q^{2} / m_{\rho}^{2} \leq 1$ (see Fig. 12).

\section{Kinematical characteristics}

As in Sec. III D, the kinematical characteristics of the coherent $\mu^{ \pm} \pi^{\mp} \pi^{0}$ events at $|t|<0.1 \mathrm{GeV}^{2}$ are studied in terms of the kinematical variables $E, \nu, Q^{2}, W, x$, and $y$ (Fig. 14), as well as the momentum transfer variables $|t|, t_{\min }$, and $t^{\prime}$ (Fig. 15). The histograms represent the
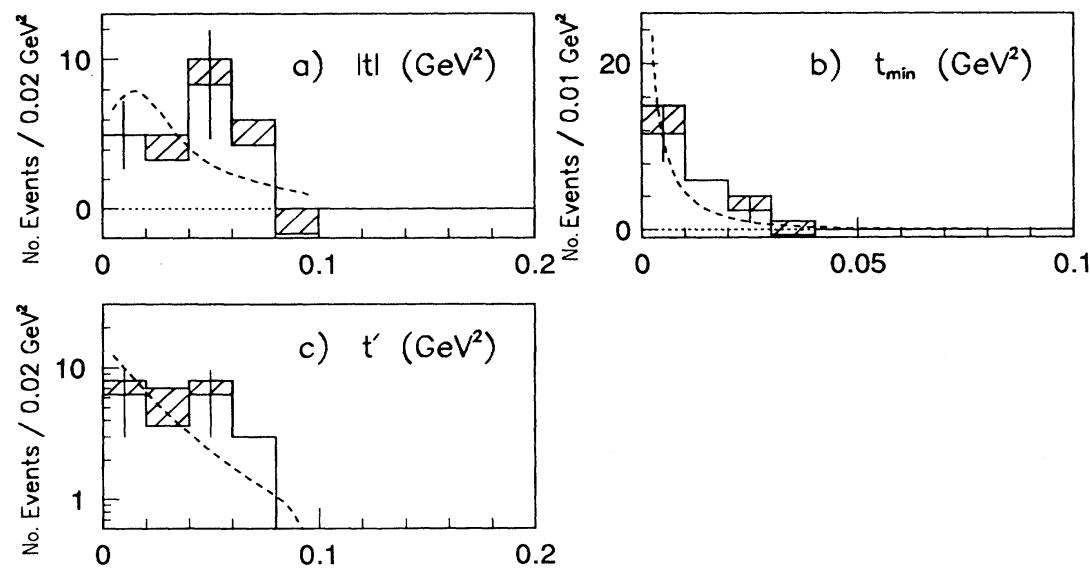

FIG. 15. Distributions of momentum transfer variables for $\mu^{ \pm} \pi^{\mp} \pi^{0}$ events at $|t|<$ $0.1 \mathrm{GeV}^{2}$ : (a) $|t|$, (b) $t_{\min }$, (c) $t^{\prime}$. The curves represent the predictions of the model normalized to the signal. The events with stubs (shown cross-hatched) have been normalized and subtracted from the distributions for events without stubs. 
TABLE VI. Average values of kinematical variables for single $\rho$ coherent events at $|t|<0.1$ $\mathrm{GeV}^{2}$. The predictions of the model are given for two parametrizations of $R$.

\begin{tabular}{lccc}
\hline \hline & Data & & Model \\
& & $R=0$ & $R=0.4 Q^{2} / m_{\rho}^{2} \leq 1$ \\
\hline$\langle E\rangle \quad(\mathrm{GeV})$ & $98.1 \pm 11.6$ & 101.1 & 102.7 \\
$\langle\nu\rangle(\mathrm{GeV})$ & $18.2 \pm 2.0$ & 29.5 & 29.5 \\
$\left\langle Q^{2}\right\rangle\left(\mathrm{GeV}^{2}\right)$ & $2.03 \pm 0.52$ & 2.18 & 2.35 \\
$\langle W\rangle\left(\mathrm{GeV}^{2}\right.$ & $5.63 \pm 0.33$ & 6.76 & 6.79 \\
$\langle x\rangle$ & $0.055 \pm 0.011$ & 0.046 & 0.051 \\
$\langle y\rangle$ & $0.249 \pm 0.032$ & 0.323 & 0.319 \\
$\langle|t|\rangle\left(\mathrm{GeV}^{2}\right)$ & $0.037 \pm 0.005$ & 0.032 & 0.033 \\
$\left\langle t_{\min }\right\rangle\left(\mathrm{GeV}^{2}\right)$ & $0.006 \pm 0.002$ & 0.007 & 0.008 \\
$\left\langle t^{\prime}\right\rangle\left(\mathrm{GeV}^{2}\right)$ & $0.031 \pm 0.005$ & 0.025 & 0.025 \\
$t^{\prime}$ slope $\left(\mathrm{GeV}^{-2}\right)$ & $24 \pm 9$ & 35 & 34 \\
$\langle\epsilon\rangle$ & $0.938 \pm 0.015$ & 0.872 & 0.879 \\
\hline \hline
\end{tabular}

distributions for events without stubs from which the normalized distributions for events with stubs (background) have been subtracted (shown cross-hatched). Also shown are the predictions of the model computed using our full Glauber simulation and with the parameter $R=0$. The predictions for $R=0.4 Q^{2} / m_{\rho}^{2} \leq 1$ differ only slightly. Similarly, the predictions obtained using the Belkov and Kopeliovich approximation do not show appreciable differences with the above. Note that there are no coherent events at $\nu \leq 2 \mathrm{GeV}$.

There is good agreement between the data and the predictions in the variables $E, Q^{2}$, and $x$, but the model predicts more events at high $\nu, W$, and $y$ than are observed. In particular, the $\nu$ distribution shows no event at $\nu>40 \mathrm{GeV}$ whereas the model predicts that there should be 5 events in that high $\nu$ region. A similar trend was reported by the WA59 and E546 Collaborations [19,20]. The detection of the second $\gamma$ of a pair might be particularly difficult if it materialized inside the shower due to its partner, and the consequent loss would preferentially affect high energy $\pi^{0}$ mesons and hence events with large $\nu$ values. However, we have not found evidence for such a loss. In particular, the average $\pi^{0}$ and $\pi^{\mp}$ momenta for the coherent events at $|t|<0.1 \mathrm{GeV}^{2}$ are nearly identical: $\left\langle p_{\pi^{\circ}}\right\rangle=(9.1 \pm 1.4) \mathrm{GeV} / c$ and $\left\langle p_{\pi \mp}\right\rangle=(9.2 \pm 1.5) \mathrm{GeV} / c$. Table VI compares the average values of the kinematical variables for the events at $|t|<0.1 \mathrm{GeV}^{2}$ with the predictions of the model for the two parametrizations of $R$.

As in the case of single pion production, the $|t|$ and $t^{\prime}$ distributions are slightly flatter than predicted. The fit of the $t^{\prime}$ distribution as an exponential gives a slope of $(24 \pm 9) \mathrm{GeV}^{-2}$, in reasonable agreement with the slope predicted for coherent interactions on neon nuclei (see Table VI).

\section{HEAVIER MESON PRODUCTION}

We have also investigated the coherent production of hadronic final states consisting of three pions. These final states are believed to be due to the production of $a_{1}$ mesons and/or $\rho \pi$ systems which decay according to

$$
\begin{aligned}
& a_{1}^{\mp} \rightarrow \rho^{\mp}+\pi^{0} \rightarrow \pi^{\mp}+\pi^{0}+\pi^{0}, \\
& a_{1}^{\mp} \rightarrow \rho^{0}+\pi^{\mp} \rightarrow \pi^{+}+\pi^{-}+\pi^{\mp} .
\end{aligned}
$$

We find 43 (28) $\mu^{ \pm} \pi^{\mp} \pi^{0} \pi^{0}, 50$ (23) $\mu^{ \pm} \pi^{\mp} \pi^{0} \gamma$, and 115 (49) $\mu^{ \pm} \pi^{\mp} \pi^{+} \pi^{-}$events without (with) stubs satisfying our selection criteria. The $\pi^{0}$ are reconstructed as described in Sec. IV A. After normalization of the $|t|$ distributions at $|t|>0.3 \mathrm{GeV}^{2}$, the signal extracted at $|t|<0.1 \mathrm{GeV}^{2}$ is determined to be $\left(7.4_{-4.0}^{+5.0}\right) \mu^{ \pm} \pi^{\mp} \pi^{0} \pi^{0}$ events, $\left(7.0_{-2.6}^{+3.5}\right) \mu^{ \pm} \pi^{\mp} \pi^{0} \gamma$ events, and (9.5 -4.1) $\mu^{ \pm} \pi^{\mp} \pi^{+} \pi^{-}$events. The $\mu^{ \pm} \pi^{\mp} \pi^{+} \pi^{-}$ events were measured only on a fraction of the film, corresponding to $77 \%$ of the total flux used in the two-prong event analysis. Because of the limited statistical significance of the signal, we have not attempted a detailed kinematical analysis of the above events.

After correction for the various cuts and losses, the overall coherent signal for $(3 \pi)^{\mp}$ final states is found to be $\left(90_{-27}^{+35}\right)$ events. The corresponding rate per charged current event is thus $\left(0.32_{-0.09}^{+0.12}\right) \%$ and the cross section, averaged over our (anti)neutrino energy spectra, is estimated to be $\left(3.2_{-0.9}^{+1.2}\right) \times 10^{-38} \mathrm{~cm}^{2}$ per neon nucleus $(E>10 \mathrm{GeV})$.

The WA59 Collaboration studied the $(3 \pi)^{-}$coherent production in antineutrino interactions, at significantly lower energy, and reported a cross section of $(0.99 \pm 0.24) \times 10^{-38} \mathrm{~cm}^{2}$ per neon nucleus for $E>15$ $\mathrm{GeV}[7]$. This measurement was found to be in reasonable agreement with the predicted cross section, given the uncertainties in the model.

\section{SUMMARY}

The coherent production of single $\pi$ and $\rho$ mesons in (anti)neutrino charged-current interactions on neon nuclei has been studied using the Fermilab 15-foot bubble chamber. The bubble chamber filled with a heavy $\mathrm{Ne}-\mathrm{H}_{2}$ mixture was exposed to the quadrupole triplet neutrino beam produced by $800 \mathrm{GeV}$ protons from the Tevatron. The average $\nu_{\mu}\left(\bar{\nu}_{\mu}\right)$ beam energy was $80 \mathrm{GeV}(70 \mathrm{GeV})$ 
and the average $\nu_{\mu}\left(\bar{\nu}_{\mu}\right)$ event energy was $150 \mathrm{GeV}(110$ $\mathrm{GeV})$.

From a sample of $250 \mu^{ \pm} \pi^{\mp}$ events, the uncorrected signal for single pion coherent production extracted from the $|t|$ distributions is found to be $(53.3 \pm 9.4)$ events at $|t|<0.1 \mathrm{GeV}^{2}$. After corrections for all cuts and identified losses, the signal is $(91.1 \pm 18.7)$ events. The corresponding number of (anti)neutrino-neon charged-current events is $(28208 \pm 682)$, yielding a production rate of $(0.32 \pm 0.07) \%$ for $\left(\nu_{\mu}+\bar{\nu}_{\mu}\right)$ interactions combined and $E>10 \mathrm{GeV}$. The single pion coherent cross section per neon nucleus is $(3.2 \pm 0.7) \times 10^{-38} \mathrm{~cm}^{2}$ averaged over our energy spectrum for $\left(\nu_{\mu}+\bar{\nu}_{\mu}\right)$ combined $(E>10 \mathrm{GeV})$. This result, as well as the separate cross sections for neutrino and antineutrino interactions, is in good agreement with the predictions of a model based on the hadron dominance and PCAC.

The kinematical characteristics of the events at $|t|<$ $0.1 \mathrm{GeV}^{2}$ and $\nu>2 \mathrm{GeV}$ were also compared with the model. Good overall agreement was obtained. The data seem to slightly favor the $\rho \pi$ cut over the $a_{1}$ pole form factor. The data thus provide a successful test of the hadron dominance and PCAC at high energy.

The coherent production of single $\rho$ mesons has been studied using a sample of $126 \mu^{ \pm} \pi^{\mp} \pi^{0}$ events, for which both $\gamma$ from the $\pi^{0}$ decay are observed and constrained to fit the $\pi^{0}$ decay hypothesis. The uncorrected coherent signal extracted from the $|t|$ distributions is $(19.2 \pm 6.8)$ events at $|t|<0.1 \mathrm{GeV}^{2}$, yielding $(59.7 \pm 23.1)$ events after corrections for cuts and losses. This corresponds to a rate of $(0.21 \pm 0.08) \%$ with respect to the total number of charged-current interactions and a cross section per neon nucleus of $(2.1 \pm 0.8) \times 10^{-38} \mathrm{~cm}^{2}$ averaged over our energy spectrum for $\left(\nu_{\mu}+\bar{\nu}_{\mu}\right)$ combined and $E>10$ $\mathrm{GeV}$.

The measured cross section is compatible with the prediction of a model based on the hadron dominance for $R=0$ but is somewhat lower than predicted for $R=0.4 Q^{2} / m_{\rho}^{2} \leq 1$, where $R$ is the ratio of longitudinal to transverse $\rho$ production. However, the angular decay characteristics provide an indication of production of longitudinally polarized $\rho$ mesons. The kinematical characteristics of the $\mu^{ \pm} \pi^{\mp} \pi^{0}$ events at $|t|<0.1 \mathrm{GeV}^{2}$ are in reasonable agreement with the model for the $E$, $Q^{2}$, and $x$ distributions, but at large values of $\nu, W$, and $y$, the number of events observed is less than predicted.

Finally, the coherent production of $(3 \pi)$ systems has been investigated in the $\mu^{ \pm} \pi^{\mp} \pi^{0} \pi^{0}, \mu^{ \pm} \pi^{\mp} \pi^{0} \gamma$, and $\mu^{ \pm} \pi^{\mp} \pi^{+} \pi^{-}$channels. After normalization of the $|t|$ distributions at $|t|>0.3 \mathrm{GeV}^{2}$, the signal at $|t|<0.1 \mathrm{GeV}^{2}$ is found to be $\left(7.4_{-4.0}^{+5.0}\right) \mu^{ \pm} \pi^{\mp} \pi^{0} \pi^{0}$ events, $\left(7.0_{-2.6}^{+3.5}\right)$ $\mu^{ \pm} \pi^{\mp} \pi^{0} \gamma$ events, and $\left(9.5_{-4.9}^{+7.1}\right) \mu^{ \pm} \pi^{\mp} \pi^{+} \pi^{-}$events. Combining these channels yields an estimated cross section per neon nucleus of $\left(3.2_{-0.9}^{+1.2}\right) \times 10^{-38} \mathrm{~cm}^{2}$, averaged over our (anti)neutrino energy spectra.

\section{ACKNOWLEDGMENTS}

We wish to thank the Fermilab staff and the 15-foot bubble chamber crew for excellent beam conditions and bubble chamber operation. We also wish to acknowledge the scanning and measuring staff in all the collaborating institutions for their careful and dedicated work. We acknowledge our colleagues from Rutherford Appleton Laboratory and CEN Saclay for their contribution to this experiment. Finally, we acknowledge B. Z. Kopeliovich for his help. This work was supported in part by the U.S. Department of Energy and the National Science Foundation, and by the U.K. Science and Energy Research Council.

\section{APPENDIX}

The differential cross section for the coherent production of single pions is given by $[1,6]$

$$
\begin{aligned}
\frac{d^{3} \sigma\left(\nu_{\mu} \mathrm{Ne} \rightarrow \mu \pi \mathrm{Ne}\right)}{d Q^{2} d \nu d k_{T}^{2}}= & \frac{G_{F}^{2}}{4 \pi^{2}} f_{\pi}^{2} \frac{|\mathbf{q}|}{E^{2}} \frac{\epsilon}{1-\epsilon} \\
& \times\left|F_{N}\left(Q^{2}\right)\right|^{2} \frac{d \sigma(\pi \mathrm{Ne} \rightarrow \pi \mathrm{Ne})}{d k_{T}^{2}}
\end{aligned}
$$

where $G_{F}$ is the Fermi coupling constant, $f_{\pi}=0.932 m_{\pi}$ is the pion decay constant, and $k_{T}^{2}$ is the square of the transverse part (with respect to the $W$-boson direction) of the momentum transfer to the nucleus. The polarization parameter $\epsilon$ is defined by

$$
\epsilon=\left(1+\frac{2|\mathbf{q}|^{2}}{4 E E_{\mu}-Q^{2}}\right)^{-1} .
$$

Following Belkov and Kopeliovich [6], the nucleon form factor $F_{N}\left(Q^{2}\right)$ may be represented by a propagator form with an axial-vector mass $m_{A}$ corresponding to the $a_{1}$ mass

$$
F_{N}^{\text {pole }}\left(Q^{2}\right)=\frac{m_{A}^{2}}{Q^{2}+m_{A}^{2}} .
$$

However, there are theoretical arguments $[1,6]$ and experimental indications [7] that the axial current may be dominated by nonresonant $\rho \pi$ systems rather than by the $a_{1}$ meson. In this case, the dispersion relation corresponding to the Regge $\rho \pi$ cut yields

$F_{N}^{\text {cut }}\left(Q^{2}\right)=\frac{\left(m_{\rho}+m_{\pi}\right)^{2}}{Q^{2}+m_{\pi}^{2}} \ln \left[1+\frac{Q^{2}+m_{\pi}^{2}}{\left(m_{\rho}+m_{\pi}\right)^{2}}\right]$.

For small $Q^{2}$, the $Q^{2}$ dependence of the pole and cut form factors is nevertheless similar [6].

The differential cross section for the coherent production of $\rho$ mesons is given by

$$
\begin{aligned}
\frac{d^{3} \sigma\left(\nu_{\mu} \mathrm{Ne} \rightarrow \mu \rho \mathrm{Ne}\right)}{d Q^{2} d \nu d k_{T}^{2}}= & \frac{G_{F}^{2}}{4 \pi^{2}} \frac{|\mathbf{q}|}{E^{2}} \frac{f_{\rho^{ \pm}}^{2}}{1-\epsilon} \frac{Q^{2}}{\left(Q^{2}+m_{\rho}^{2}\right)^{2}} \\
& \times(1+\epsilon R) \frac{d \sigma_{T}(\rho \mathrm{Ne} \rightarrow \rho \mathrm{Ne})}{d k_{T}^{2}}
\end{aligned}
$$

with the ratio between longitudinal and transverse $\rho$ nucleus cross sections:

$$
R=\frac{\sigma_{L}(\rho \mathrm{Ne} \rightarrow \rho \mathrm{Ne})}{\sigma_{T}(\rho \mathrm{Ne} \rightarrow \rho \mathrm{Ne})} .
$$


The value of $R$ is not known experimentally. Suggested parametrizations of $R$, based on $\rho^{0}$ production in electron and muon interactions, are $R=0$ or $R=0.4 Q^{2} / m_{\rho}^{2} \leq 1$ [33].

The coupling constant to the $W$ boson, $f_{\rho^{ \pm}}$, is given in terms of $f_{\rho^{0}}$, the $\rho^{0}$ coupling constant to the photon: $f_{\rho^{ \pm}}=f_{\rho^{\circ}} \sqrt{2} \cos \theta_{C}$, where $\theta_{C}$ is the Cabibbo angle, $f_{\rho^{\mathrm{o}}}=m_{\rho}^{2} / \gamma_{\rho}$ and $\gamma_{\rho}^{2} / 4 \pi=(2.4 \pm 0.1)$. The value of $\gamma_{\rho}^{2} / 4 \pi$ is determined by using $\tau \rightarrow \rho \nu_{\tau}$ decay data [34] and the narrow resonance approximation [35]:

$\Gamma\left(\tau \rightarrow \rho \nu_{\tau}\right)=\frac{G_{F}^{2}}{8 \pi} \frac{V_{u d}^{2}}{\gamma_{\rho}^{2}} \frac{m_{\rho}^{2}}{m_{\tau}^{3}}\left(m_{\tau}^{2}+2 m_{\rho}^{2}\right)\left(m_{\tau}^{2}-m_{\rho}^{2}\right)^{2}$.

Using the Glauber model, we write the hadron-nucleus elastic scattering cross section as

$$
\frac{d \sigma(h \mathrm{Ne} \rightarrow h \mathrm{Ne})}{d k_{T}^{2}}=\frac{\left(\sigma_{\mathrm{tot}}^{h N}\right)^{2}}{16 \pi}\left(1+r^{2}\right)|g|^{2},
$$

where $r$ is the ratio between the imaginary and real parts of the forward scattering amplitude $(r=-0.2)$. The function $g$ is expressed as

$$
\begin{aligned}
g(\mathbf{k})=\int d^{2} b \int & d z e^{i \mathbf{k}_{T} \cdot \mathbf{b}} e^{i k_{L} z} \rho_{\mathcal{N}}(\mathbf{b}, z) \\
& \times e^{-\frac{1}{2} \sigma_{\text {tot }}^{\pi N} \int_{-\infty}^{z} d z^{\prime} \rho_{\mathcal{N}}\left(\mathbf{b}, z^{\prime}\right)},
\end{aligned}
$$

where $\mathbf{b}$ is the impact parameter in the plane perpendicular to the hadron direction and $z$ is the coordinate along its direction; $k_{L}$ is the longitudinal part of the momentum transfer to the nucleus. At high energy and small $|t|$, we have $k_{L}^{2} \simeq t_{\min }$, and $k_{T}^{2} \simeq t^{\prime}=|t|-t_{\min }$. The pion-nucleon cross section $\sigma_{\text {tot }}^{\pi N}$ is taken at the energy $E_{\pi}=\nu$. The nuclear density function is represented by the Woods-Saxon form

$$
\begin{aligned}
\rho_{\mathcal{N}}(r) & =\frac{\rho_{0}}{1+e^{\left(r-R_{\mathcal{N}}\right) / a}}, \\
\rho_{0} & =\frac{3 A}{4 \pi R_{\mathcal{N}}^{3}} \frac{1}{1+\pi^{2} a^{2} / R_{\mathcal{N}}^{2}},
\end{aligned}
$$

with $R_{\mathcal{N}}=2.80 \mathrm{fm}$ and $a=0.571 \mathrm{fm}$ for neon [36].

In the case of $\rho$ production, we have taken the $\rho$ nucleon cross section to be the same as the $\pi$-nucleon cross section because of the lack of direct measurement.

Belkov and Kopeliovich have approximated the hadron-nucleus elastic scattering cross section given in Eqs. (A4) and (A5), as the product of two exponentials $[6]$ :

$\frac{d \sigma(h \mathrm{Ne} \rightarrow h \mathrm{Ne})}{d k_{T}^{2}}=\frac{\left(\sigma_{\mathrm{tot}}^{h \mathrm{Ne}}\right)^{2}}{16 \pi}\left(1+r^{2}\right) e^{-B_{T} k_{T}^{2}} e^{-B_{L} k_{L}^{2}}$

The slope parameters $B_{L}$ and $B_{T}$ have been computed according to Ref. [6]. For neon and $\sigma_{\text {tot }}^{\pi N}=24 \mathrm{mb}$, the slopes are found to be $B_{L}=75.8 \mathrm{GeV}^{-2}$ and $B_{T}=88.2$ $\mathrm{GeV}^{-2}$, whereas the total pion-nucleus cross section is found to be $\sigma_{\text {tot }}^{\pi \mathrm{Ne}}=372 \mathrm{mb}[23]$.
[1] B. Z. Kopeliovich and P. Marage, Int. J. Mod. Phys. A 8, 1513 (1993).

[2] WA21 Collaboration, G. T. Jones et al., Z. Phys. C 37, 25 (1987).

[3] S. L. Adler, Phys. Rev. 135, B963 (1964).

[4] C. A. Piketty and L. Stodolsky, Nucl. Phys. B15, 571 (1970).

[5] See, for instance, T. H. Bauer, R. D. Spital, D. R. Yennie, and F. M. Pipkin, Rev. Mod. Phys. 50, 261 (1978), and references therein.

[6] A. A. Belkov and B. Z. Kopeliovich, Yad. Fiz. 46, 874 (1987) [Sov. J. Nucl. Phys. 46, 499 (1987)].

[7] WA59 Collaboration, P. Marage et al., Z. Phys. C 49, 385 (1991).

[8] R. T. Deck, Phys. Rev. Lett. 13, 169 (1964).

[9] J. S. Bell, in Hadronic Interactions of Electrons and Photons, edited by J. Cumming and H. Osborn (Academic, London, 1971), p. 369.

[10] E632 Collaboration, M. Aderholz et al., Phys. Rev. Lett. 63, 2349 (1989).

[11] Aachen-Padova Collaboration, H. Faissner et al., Phys. Lett. 125B, 230 (1983).

[12] E. Isiksal, D. Rein, and J. G. Morfin, Phys. Rev. Lett. 52, 1096 (1984).

[13] CHARM Collaboration, F. Bergsma et al., Phys. Lett. 157B, 469 (1985).

[14] WA59 Collaboration, P. Marage et al., Z. Phys. C 31, 191 (1986).

[15] SKAT Collaboration, H. J. Grabosch et al., Z. Phys. C
31, 203 (1986).

[16] E53 Collaboration, C. Baltay et al., Phys. Rev. Lett. 57, 2629 (1986).

[17] E180 Collaboration, V. V. Ammosov et al., Yad. Fiz. 45, 1662 (1987) [Sov. J. Nucl. Phys. 45, 1029 (1987)].

[18] WA59 Collaboration, P. Marage et al., Z. Phys. C 43, 523 (1989).

[19] WA59 Collaboration, P. Marage et al., Z. Phys. C 35, 275 (1987).

[20] E546 Collaboration, H. C. Ballagh et al., Phys. Rev. D 37, 1744 (1988)

[21] R. J. Glauber, in Lectures in Theoretical Physics, edited by W. E. Brittin and L. G. Dunham (Interscience, New York, 1959), p. 315.

[22] A description of the Fermilab neutrino flux program (NUADA) can be found in D. C. Carey and V. A. White, Fermilab Computing Division Report No. PM0011, 1975 (unpublished). For more details about the neutrino beam see L. G. Stutte, Fermilab Technical Memo No. TM-1305, 1985.

[23] S. Willocq, Ph.D. thesis, Tufts University, 1992.

[24] E632 Collaboration, V. Jain et al., Phys. Rev. D 41, 2057 (1990).

[25] WA59 Collaboration, P. Marage et al., Phys. Lett. 140B, 137 (1984).

[26] CCFR Collaboration, P. S. Auchincloss et al., Z. Phys. C 48, 411 (1990).

[27] The predictions of the model proposed by D. Rein and L. M. Sehgal, Nucl. Phys. B223, 29 (1983), which uses 
a crude approach to the pion absorption inside the neon nucleus, do not differ greatly from those of the model used in this analysis.

[28] ARGUS Collaboration, H. Albrecht et al., Z. Phys. C 33, 7 (1986).

[29] See, for instance, D. W. G. S. Leith, in Electromagnetic Interactions of Hadrons, edited by A. Donnachie and G. Shaw (Plenum, New York, 1978), Vol. I, p. 345.

[30] M. Ross and L. Stodolsky, Phys. Rev. 149, 1172 (1966).
[31] J. Lys (private communication)

[32] K. Schilling and G. Wolf, Nucl. Phys. B61, 381 (1973).

[33] See Ref. [1] and references therein.

[34] Particle Data Group, K. Hikasa et al., Phys. Rev. D 45, S1 (1992).

[35] L. B. Okun, Leptons and Quarks (North-Holland, Amsterdam, 1982).

[36] H. DeVries, C. W. DeJager, and C. DeVries, At. Data Nucl. Data Tables 36, 495 (1987). 